\title{
Wann sollen wir rachitische Verkriimmnngen gerade richten?
}

\author{
Fon \\ Sau.-Rat Gangele. \\ (Aus der Orthopädischen Heilanstalt und dem Krüpelhein Zwickau, Sa.)
}

Siit 3 Abbildungen in Text.

(Eingegangen am 11. Januter 1922.)

Bis ror kurzem war ich der Ansicht, daß dlese Frage lingst und in klarer Weise beantwortet sei. Gaben uns doch unsere Meister, wie Hoff a und Lange, aus ihrer großen Etfahrung heraus den Rat, der Spontanheilung rachitischer Verkrümmungen nicht allzusehr zu rertrauen, rielmehr zeitig einzugreifen. Auch die treffliche Arbeit ron $\mathrm{KrauB}$ jun. in Brl. $26 \mathrm{~d}$. Teitschr. f. orthop. (hirurg., in welcher sich lieser auf denselben Stanrlpunkt stellt. blieb in den nachfolgenden Zeiten ohne Widerspruch.

Ich glaubte, der Satz, man solle die rachitischen Verkr ümmungen erst in 6. Lebensjahr und darnach angreifen. sei nur noch in alten Lehrbüchern der Nichtorthopäden zu finden, glaubte auch, daß diesem satze nur noch wenige älere Kollegen cor allgemeinen Praxis hulligten. Ich nahm an, die Geschichte der Orthopädie in den letzten 20 Jahren hätte zur Genügt dargelegt, daß wir jerle MiBbildung des menschlichen Körpers in Frühstadium angreifen sollen. Krau B sagt in genannter Arbeit so schön: .. Was würcle man wohl von einem Gärtner vagen, der alte Bäume gerarle richten wollte, nachlem er vorher vergebens ihre spontane Geradstrechung abgewartet hätte? Jeder Gärtner setzt neben einen gekrummten jungen Baum eine Stiitze und bindet ihn an, dann wächst er sich ron selbst gerade."

Die Mehrzahl der Orthopäden denkt ja wohl hier genau so wie Krauß und ich. Wenn aber auf dem diesjährigen Orthopädenkongreß ein angesehener Fachkollege einer Verzögerung der aktiven Inangriffnahme rachitischer Verkrümmungen das Wort redete, so dürfte es doch nötig sein, in unseren Fachzcitschriften diese Frage nochmals aufzurollen. Es wäre durchaus verdienstvoll und äußerst wichtig für unser Fach, wenn zu dieser Frage auch andere Kollegen das Wort ergreifen und ihre Erfahrungen mitteilen würden.

Meine Erfahrungen sprechen gegen jede Verzögerung der redressierenden Behandlung, gegen jedes Zuwarten. Ich halte jeden 
zum Zuwarten ratenden Vorschlag für unangebracht; denn meines Erachtens ist er geeignet, die Vorbedingungen der Erfolge unserer Rachitisbehandlung zu zerstören, indem er die Kollegen der allgemeinen Praxis wieder zu abwartendem Verhalten veranlaßst und dadurch häufig die Ursache für ein ,,zu spät" werden wird. Jedenfalls düufen derartige Vorschläge nur ganz selten Gehör finden; sie können nur die Ausnahme der Regel bilden.

Ich will mich nun im folgenden bemühen, objektiv die Vorzüge und Nachteile der „Früh- und Spät.Geradrichtung" zu beleuchten und meinen schon eben angegebenen Standpunkt durch Beispiele zu bekräftigen suchen.

In der Literatur sind es hauptsächlich zwei Arbeiten, auf welchen sich dit These, rachitische Verkrümmungen nicht vor dem 6. Jahr anzugreifen, aufgebaut hat. Beide stammen aus dem Jahre 1895. Veit, Über die Spontanheilung von rachitischen Verkrümmungen; in Langenbecks Arch., Bd. 50 und Kamps, Uber die spontane Geradstreckung der rahitischen Unterschenkelverkrümmungen; Bruns Beitr. z. klin. Chirurg. Brl. 14. Letzterer sebildert
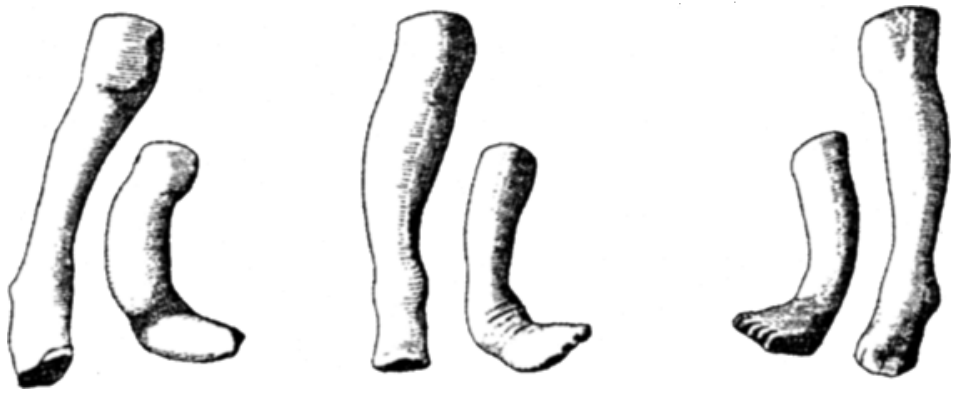

Abb. 1 .

die Erfahrungen aus der Tübinger chirurgischen Klinik, Eısterer aus der chirurgischen Universitäts-Poliklinik Berlin. Ka mps hat bei Beginn der Behandlung und nach Abschluß der Behandlung Gipsmodelle gemacht. Die durchschnittliche Beobachtungszeit betrug $4 \frac{1}{2}$ Jahre. Angeblich handelt es sich nur um ganz schwere Fälle, wobei er $75 \%$ Heilung beobachtet haben will. Einige seiner Gipsmodelle sind in Zeichnungen (Photographien?) wiedergegeben. Man darf ja nun wohl annehmen, daß er zur Illustration nicht et wa seine schlechtesten Fälle veröffentlicht hat. Hohmann hat die Abbildungen auf dem diesjährigen Orthopädenkongre $\beta$ gezeigt, und ich bringe hier einen Teil seiner Zeichnungen wieder (Abb. 1). Es mutet uns heute, nach 25 Jahren, eigentümlich an. wie Verfasser ron ,Heilungen" der Ver kr ümmungen in seinen Fällen sprechen konnte. Denkt man sich die irreführende Klumpfußstellung des Gipsmodells bei Beginn der Behandlung weg, dann müssen wir konstatieren, daß die Endresultate sich herzlich wenig von den ersten Aufnahmen unterscheiden. Auf jeden Fall sind noch O-Beine da. Wenn wir mit solchen Resultaten, für die alle Orthopäden nur ein mitleidiges Lächeln haben werden, zufrieden sein sollen. dann könnten wir allerdings auf eine frühzeitige Geradrichtung verzichten. Unsere Kol. legen der allgemeinen Praxis und vor allem auch das Publikum wollen aber heutzutage von uns andere Erfolge; sie verlangen von uns mit Recht, da $B$ wir gerade Glieder schaffen und wärden sich nicht zufrieden geben, wenn unsere 
Patienten nach jahrelanger Behandlung immer noch O-Beine haben. Wenn die Orthopärlie in den letzten 20 Jahren einen so gewaltigen Aufschwung genommen hat, so doch hauptsächlich deswegen, weil wir dem praktischen Arzte und dem Publikum in die Augen fallende Resultate bei unserer Geraderichtungen zeigen konnten. In einem gewissen Gegensatz zu $K a m p s$ hat Veit uns tatsichlich gezeigt. daß auch schwere Verkrümmungen ohne redressierende Behand. lung gerade werden können. Wenigstens kann man dies von den zwei ersten Fallen Veits sagen. Auch Schanz und andere haben uns ja nachgewiesen, raßs Spontanheilungen vorkommen; Schanz allerdings mit der sofortigen Eirshränkung, daß eine Spontanheilung nur bei mäßigen Deformitäten und im fr ühe'sten Kindesalter zu erwarten sei. Veit selbst bringt eine Einschränkung; e' gibt an, daß manche Fälle ron O-Beinen eine ganz ungünstige Prognose biete'n. Als Resultat seiner Untersuchung hören wir: „Alle, auch schwere Fälle von rachitisehen Verkrümmungen der Gliedmaßen können spontan sich strecken, wenn die Tendenz zum Wachstum resp. zur Entwioklung des ganzen Skelettes besteht."

Meine Erfahrungen aus einer $16 \mathrm{jährigen} \mathrm{Tätigkeit} \mathrm{in} \mathrm{Zwickau}$ lehren mich anderes. Zunärhst habe ich of Gelegenheit genommen, die Angaben von Müttern über spontane Heilungen nachzuprüfen. Das Spiel war jedesmal dasselbe: Zuerst erzählen uns die Mütter von vollkommenen Heilungen. Fragt man genauer, so erfährt man, daß eine leichte Schwingung schon noch zu sthen sei. Noch unsicherer werden die Wütter, wemn ich sie auffordere, ihre Kinder nitzubringen, damit ich mich selbst von dem schönen Resultat überzengen könne. Sieht man die Kinder endlich vor sich, so sehen die „Heilungen" meist so aus, wie sie uns Kamps gezeigt hat.

Theoretische Erwägungen sowohl wie die Erfahrungen der Praxis sprechen tür Frühredressement.

1. Die Zeit des Wachstums der einzelnen Knochen ist eine ganz verschitrene. So wächst die Wirbelsäule nach Engel mann hauptsächlich in den ersten 3 Jahren; zwischen dem 3. und 6. Lebensjahr ruht das Wachstum fast voll. ständig. Ist e's erlaubt, die Zeit de's besten Ausgleiches hipr verstreichen zu lassen? Denn wenn wir voraussetzen - und die Erfahrungen bestiitigen us daß wir durch unsere korrigierenden Maßnahmen (ev. auch ohne Osteotomie und Osteoklasie) noch eine Knochenumformung erreichen können, so können wir es am besten zur Zeit der größten Weichheit der Knochen. Diese ist aber' beim wachsenden Knochen viel größer als beim nichtwachsenden. So sind auch Schädigungen in den Jahren rlex stärksten Wachstums besonders gefährlich, weil der warhsende Knochen infolge seiner Weichheit abnormen Einflüssen, ja selhst schon der Belastung mehr nachgeben wird als der nichtwachsende Knochen. Das wichtigste aber ist, daß die während der blühenden Rachitis entstandenen Wachtumsbeeinträchtigungen zu einem großen Teil in der nachrachitischen Wachstumsperiode schon nicht mehr ausgeglichen werden können. Engel mann hat dies für die rachitischen Veränrlerungen an den Wirbelbogenfugen nachgewiesen. Ein jeder weiß ja auch, daß die scheußlichsten Skoliosen aus der Zeit der floriden Rachitis stammen; ein jeder weiß ferner, wie bald gerade bei der rachitischen Skoliose des frühesten Kindesalters die Zeit des Eingreifens versäumt ist. Im allgemeinen kann man sagen, daß ein solehes Versäumnis 
dann eintritt, wenn man die korrigierenden Maßnahmen nicht zur Zeit der blühenden Rachitis, also des weichen Knochenstarliums vornimnit. Oft schon hat der Säugling eine bereits ziemlich stark fixierte Skoliose, dif nan nur mit kräftige'm Handdruck gerarle noch ausgleichen kann. Das gleiche erreichen wir mit dem Korsett, wenn auch langsamer. Erfolgt keine Korrektur, so kann uns die Besserung der Knochenfestigung unangenehm überraschen, und die allgemeine Besserung der Rachitis, die wir ja anstreben. bringt anstatt des gehofften Ausgleiches der Verbiegung die Fixierung der in bereits mißbildeter Form festgewordenen Knochen und damit ihre dauernde Unheilbarkeit. Darüber sind wir uns ja alle klar: eine sklernsierte rachitische Skoliose heilen wir nie mehr, auch nicht clurch Abott.

2. Die englische Krankheit befallt nicht alle Knochen des Körpers gleichzeitig. Nach Engel mann werten Schädel, Wirbelsäule und Brustkorb im ersten halben Jahr der Krankheit befallen. Es war mir bisher nicht möglich. diese Angabe genau nachzuprüfen. Sicher ist sie of riehtig. Ebensooft scheint mir aber auch umgekehrt die Rachitis der Beine früher oder wenigstens. schärfer einzusetzen als am Brustkorb. In unserem Krüppelheim Zwickau-Marienthal mit unserem großen rachitischen Material, wo wir auch zahlreiche Säuglinge und Kinder im 2. Lebensjahr mit Rachitis ohne Verkrümmungen aufnehmen, sehe ich ebenso häufig, , $R$ u m p f rachitiker" (Abb. 2) wie "Beinrachitiker"1). Es ist oft geradezu auffallend, wie bei einem krummen. schlangenförmigen Bein die Wirbelsäule ganz gerade getblieben ist, ja nicht einmal der Froschbauch ist immer vorhanden. und ebenso sehen wir umgekehrt ganz. schwere Skoliosen ohne Verkrümmungen der Beine. Dieses "lokalisierte Auftreten" rer Rachitis ist meines Erachten. nach nicht einwandfrei erklärt. Man kann sich vielleicht die Sache so vorstellen, daß eine bislang unbehandelte Rachitis mit Verbiegungen am Rumpf oler an den Beinen durch eine entrgisch einsetzenrle Behandlung so rasch

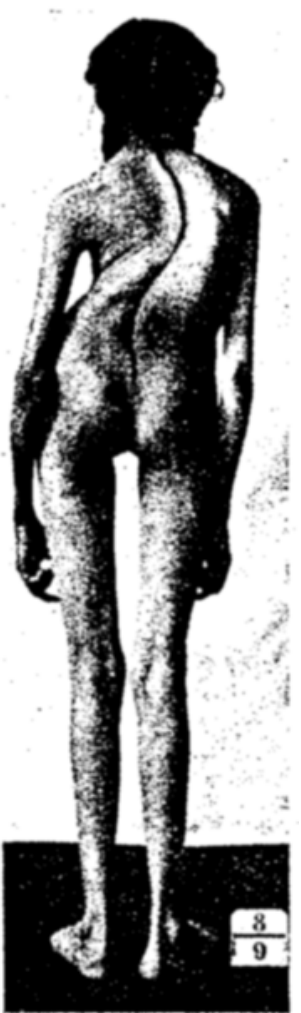

Abb. 2 . gebessert wird, daß es zu Verbiegungen der anderen Gliedmaßen nicht mehr kommt. In diesem Falle besteht aber, wie unter Nr. 1 angegeben, eine gewisse Gefahr darin, daß die rasch einsetzende Besserung eine zu rasche Sklerosierung der Knochen bringt, anstatt den Ausgleich rler Verbiegung zu fördern.

3. Preiser hat auf die Gefahren einer falschen statischen Belastung hingewiesen. Es ist sicher nicht gleichgültig, ob ein rachitisches Kind mit krummen Beinen jahrelang falsch auftritt. Am schlimmsten müssen die Mißverhältnisse einer falschen Statik in solchen Fällen zutage treten, bei denen

1) Ich verweise hier auf die Diskussionsbemerkingen Lanyes in der sitzung des Ärztichen Vereins München vom 23. XI. 21 (Münch. med. Wochenschr. Nr. l) über die Lokalisation der rachitischen Verkrümmungen. 
direkt oberhalb der Fußknörhel die Achse winklig abgeknickt ist. Daß alle O-Beine von allein gerade werden, wird ja wohl niemand behaupten: Die senkrecht zur Frde wirkende Körperlast muß naturgemäß eher eine weitere Versehlechterung zur Folge haben als eine Besserung. Wer dif Röntgenplatten seiner Rachitiker durchsieht, wird staunen, in wie vielen Fällen er bereits eine cleformierende Arthritis an den Gelenkflächen nachweisen kann, besonders häufig beim $X$-Bein. Selbst wenn wir sonst gar keinen Grund für eine früh:zeitige Gerallichtung der rachitischen Verkrümmungen hätten, die Tatsache der arthritischen Veränderungen müßte uns allein schon zur frühzeitigen Behandlung zwingen.

4. Die falsche Statik wird auch einen ungünstigen Einfluß auf die Wachstumszonen haben müssen. Ich verweise auf die $\mathrm{Abb}, 3 \mathrm{a}, \mathrm{b}, \mathrm{c}$. Wir sehern.

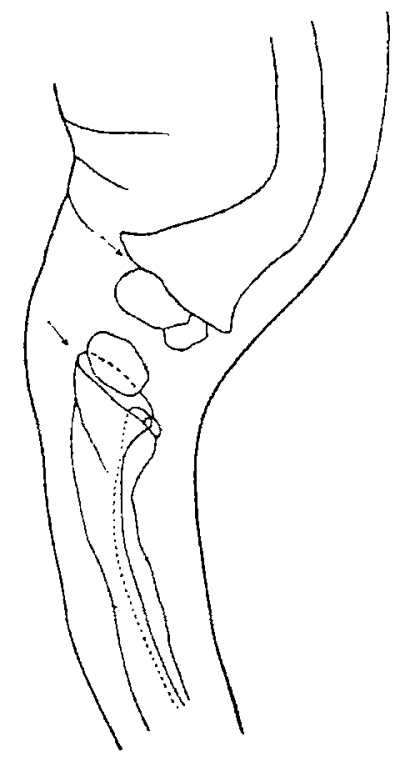

Abb. $3 a$.

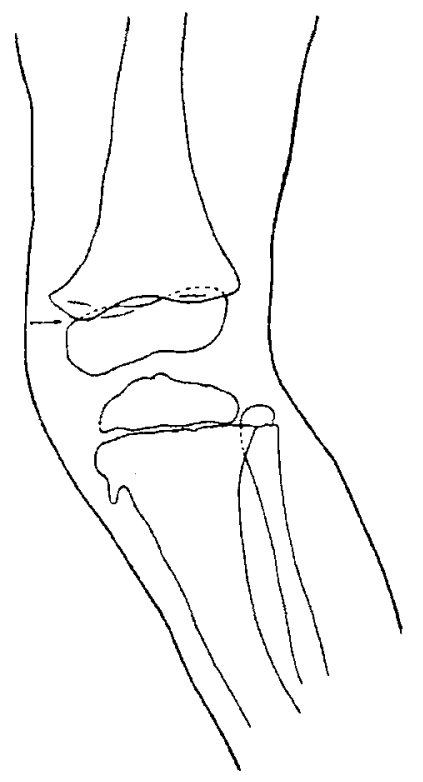

Abb. $3 b$.

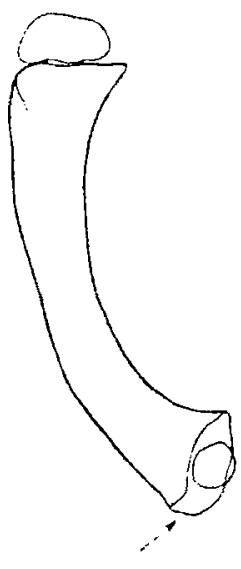

Abb. 3c.

daß die Wachstumslinien an der Tibia (Abb. $3 \mathrm{c}$ ) nahezu senkrecht steht anstatt wagrecht. Kaum eine Epiphysenlinie liauft normal. Glaubt jemand, dab eine derartige Verschiebung der Epiphysenlinie ohne Einfluß auf das Wachs. tum sein kann? Sollen wir da wirklich zuwarten, wo wir die leichte Möglichkejt haben, der Wachstumslinie ihre normale Richtung wieder zu verschaffen? Diese Verschiebung der Wachstumslinie birgt den größten Scharten in sich. sie bildet die Ursache für eine dauernde Schädigung des Längenwachstu ms. Eigentlich sollte man darüber nicht viel Worte verlieren. Der rachitische Zwergwuchs ist doch bekannt. Daß eine gekrünmte Wirbelsäule kürzer ist als eine gerade, wird auch jeder zugeben. Es ist aber falsch anzunehmen, da $B$ die Verkürzung nur so viel betrage, als die Verbiegung ausmacht. Auch hier sind die Zwergwüchsler das beste Beispiel. Wie oft haben sie einen ganz normal gebauten oder gar einen überkräfrigen Rumpf und dabei abnorm kurze Beine. 
Hier kommt "lie ,lokalisierte Rachitis" so richtig zum Ausduck. Selbstverständlich können auch Rachitiker groß werden, sobald die Krankheit ausgeheilt ist. Ich behaupte aber, daß alle stärkeren Verkrümmungen des Körpers, besonders der Beine, ein dauerndes Manko des Längenwachstums herbeiführen, wenn die Verkrümmungen nicht im blühenden Stadium ausgeglichen werden. Dem widerspricht nicht. die Ansicht von Brandenburg, der auch später, offenbar in sklerosierten Stadium, nach erfolgter Osteotomie eine Zunahme des Wachstums bemerkte. Auch ich habe diese Erfahrung gemacht, aber das Wachstum ist kein so ausgiebiges, als wenn die Geraderichtung im blühenden Stadium erfolgt. Wenn Veit schreibt, daß alle, auch schwere Fälle von rachitisch»n Verkrümmungen der Gliedmaßen, spontan sich strecken, wenn die Tendenz zum Wachstum resp. zur Entwicklung des ganzen Skelettes besteh", so ist er meines Erachtens einem grundsätzlichen Irrtum zum Opfer gefallen. Das Wesen der englischen Krankheit besteht ja eben darin, daß die Tendenz zum Wachstum fehlt. Wachsen dite Kinder wierler besser, dann ist eben die Krankheit durch zweckentsprechende Behandlung gebessert worden. Wenn Veit ferner sagt, daß alle solche Fälle dann heilen, wenn die Kinder mindestens die Durchschnittsgröße ihres Alters erreichen oder darüber. so kann man das schon eher unterschreiben. Aber leider ist dies sehr selten der Fall. Ioh habo aus den Pfleglingen des Krüppelheims 18 beliebige rachitische Kinder in ver. schiedenem Lebensalter ausgewählt und gemessen. Fon diesen 18 Kindern zeigt nur ein einziges Kind die Durchschnittsgröße seines Alters. Alle anderen sind kleiner, zum Teil hochgradig kleiner und ein Utberblick der beifolgenden Liste zeigt, daß die Mankozahlen mit zunehmendem Alter um so größer werden (in der Liste bedeutet die Zahl in Klammern die normale Durchschnittsgröße. die Schlußzahl die fehlende Größe):

\begin{tabular}{|c|c|c|c|c|c|c|c|c|c|c|}
\hline Hunger & - & $\left.m \cdot{ }^{1}\right)$ & 2 & Jahre & 85 & $\mathrm{~cm}$ & $(85$ & $(\mathrm{cm})$ & 0 & $\mathrm{~cm}$ \\
\hline Götzel & . & $\left.w^{2}{ }^{2}\right)$ & 2 & $s:$ & 77 & ,. & 184 & .1 & 7 & , \\
\hline Hückel & . & $\mathrm{m}$ & 3 &, & 90 & ,. & $(93$ &, 1 & 3 & ., \\
\hline Fischer & . & $\mathrm{m}$ & 4 & $\therefore$ & 88 & , & $\langle 99$ & $.)$. & 11 & , \\
\hline Müller & . & $\mathrm{m}$ & 4 & $\because$ & 81.5 & , & (99 & $,:)$ & 17.5 & .. \\
\hline Fischer & . & $w$ & 4 & .. & 88.5 & $\because$ & 198 & )$. & 9.5 & $\because$ \\
\hline Erler . & . & $w$ & 4 & .. & 75 & : & 198 & $,)$, & 23 & ,. \\
\hline Hösel . & . & in. & 5 & ,. & 96 & ., & $(104$ &, ) & 8 & $\because$ \\
\hline Prüfer & . & $\mathrm{m}$. & $\tilde{5}$ & , & 84 & ", & (104 & ,. ) & 20 & :. \\
\hline Röhn . & . & $w$ & 8 & :. & 91 & ", & (118 & $.)$, & 27 & .. \\
\hline Krämer . . & . & $w$. & 8 & .. & 91,5 & ", & (118 & .,) & 26,5 &, \\
\hline Pechmann & . & $\mathrm{m}$. & 9 & , & 119 & ,. & (125 & ,. ) & 6 & , \\
\hline Geisler . . & . & $\mathrm{m}$. & 9 & $\therefore$ & $108, \overline{0}$ & ", & (125) & ,") & 16,5 & \\
\hline Hohenstein & . & $w$ & 12 & , & 88 & ., & (139 &, ) & 51 & $\because$ \\
\hline Fischer . & . & $w$. & 12 & $\because$ & 124 & , & (139 &, ) & 15 & , \\
\hline Singer . & . & $w$ & 12 & , & 95 & ,. & (139 &, ) & 44 & ,. \\
\hline Enderlein & . & $w$. & 17 & ,. & 145 & $\because$ & (161) & ,. ) & 16 & , \\
\hline Schenkel & $\cdot$ & $w$. & 18 & , & 124 & , & (161 &,, ) & 37 & ' \\
\hline
\end{tabular}


Rachitische Kinder, welche kein Manko an Lüngenwachstum erleiden, gibt es sicher nur in verschwindend geringer Zahl.

Uberblicken wir die Vorteile einer frühzeitigen Behandlung, so sehen wir. daß wir, sei es durch Schienenbehandlung, sei es durch ein forziertes Vorgehen, wie Osteoklasie, die Verkrümmungen sowohl am Rücken wie an den Beinen und Armen im floriden Stadium ausgleichen können. Selbstverständlich muB gleichzeitig eine energische antirachitische Allgemeinbehandlung einsetzen, und dann glaube ich, können wir heute mit einer nahezu an Sicherheit grenzenden Wahrseheinlichkeit voraussagen, daß wir rachitische Schädigungen der späteren Jahre ein für allemal verhüten können.

Welche Gegengründe lassen sich nun gegen das Frühredressement anführen:

a) Veit sagt scheinbar logisch ganz richtig: ,Warum sollen wir ein Glied er. sogar chirurgisch angreifen, wenn es die Natur von selber wieder geraderichtet." Wenn dem so wäre, hätte er Recht. Daß es aber nicht der Fall ist, zeigen uns die Krüppelheime.

b) Noeggerath empfiehlt die Behandlung der in ihren Verkrümmungen erstarrten Glieder wegen der Pneumoniegefahr nicht ror dem 5. Lebensjahr. Ich habe nie eine Pneumonie bei meinen Geradrichtungen, die wohl weit in die Tausende gehen, gesehen. Bei einem Säugling, wo die Gefahr am größten wäre, kommen wir beinahe doch stets mit Schienchen aus. Im 2 . und 3 . Lebensjahr ist die Gefahr einer Pneumonie, sowie die Gefahr der Narkose an sich. nur noch gering.

c) Etwas anderes ist es mit der Fettembolie. Ich habe tatsächlich in einem Fall nach der Geradrichtung eines X-Beines eine ganz bedrohlich auftretende Fettembolie erlebt. Aber einnal ist diese Erscheinung eine ganz verschwindend seltene und andererseits sind wir heute durch forzierte Kochsalzinjektionen tatsächlich in der Lage, jede rechtzeitig erkannte Fettembolie in kürzester Zeit zu beheben.

d) Als Student lernte ich, man solle die rachitischen Verkrümmungen deswegen erst nach dem 8 . Lebensjahr angreifen, weil man dann kein Rezidiv zu befürchten brauche. Dieser Einwurf kann heute im Ernst nicht mehr gemacht werden. Denn wenn wir, wie wir das ja alle tun, in der Nachbehandlung ein leichtes Schienchen oder bei der Skoliose ein Korsett tragen lassen, dann sehen wir eben kein Rezidiv. Ich habe noch keines gesehen. Auch ist die Technik der Bandagenfabrikation heute doch so fortgeschritten, daß sie wohl überall ausreichende Stützen schaffen kann.

Ein sehr wichtiger Punkt spricht aber noch für Frühredressement: Der Kostenpunkt. Wenn ein Arzt in der Praxis aurea einen Säugling oder ein nur wenig älteres Kind, das dank der günstigen Lebensbedingungen auch nur eine geringe Rachitis aufweist, abwartend behandelt, so ist dies selbstverständlich zu billigen, um so mehr, als in der Praxis aurea gröbere Verkrümmungen wahrscheinlich selten da sein werden. Ganz anderes verlangt aber dic Praxis paupera, zumal die Praxis unseres heute so gänzlich verarmten Mittelstandes. Hier ist keine Möglichkeit abzuwarten, denn jedes Abwarten vergrößert die Kosten. Die wenigen Fälle der Praxis a urea 
dürfen und können bei Festsetzung einer Normbehaudlung nicht maßgebend sein.

Unter "Geraderichten" verstehe ich nicht ohne weit"res die Geraderichtung durch Osteoklasie oder Osteotomie. Auch das länger wirkende Redressement, wie wir es in unseren Nachtschienen, Tauschienen, Korsetts usw. haben, genügt in zahlreichen Fällen. Wenn $\mathrm{Krau} B$ in oben genannter Arbeit die orthopädische Schiene als das souveräne Heilmittel der rachitischen Verkrümmungen bezeichnet, so kann ich ihm hierin wohl beipflichten, wenn ich auch in den Kriegsjahren und darnach mehr und mehr aus wirtschaftlichen Gründen zur möglichst rarlikalen Frühbehandlung übergegangen bin. Ich sehe keinen wichtigen Grund, der mich z. B. hinderte, bei einem rachitischen Kinde von $3 / 4-11 / 2$ Jahren, im Starium der blühenden Rachitis ein O-Beinchen durch Einknicken der Knochen geradezurichten. Das geht so schnell, tut den Kindern kaum weh: und hat, wenn man die Sache ohne Narkose macht, gar keine unangenehmen Folgen.

Ehe ich am Schlusse dieser Arbeit eine Aufstellung der Behandlungsmethoden, wie ich sie seit Jahren übe, angebe, möchte ich ausdrücklich betonen, ¿laß ich mir diese Aufstellung in einer orthopädischen Fachzeitschrift nur deswegen erlaube, weil ich sämtliche Methoden, vom ultrakonservativen Abwarten bis zur radikal-chirurgischen Frühoperation im Laufe meiner 16 jährigen Tätigkeit hier durchgeprobt habe. Selbstverständlich wird die Behandlung für seltent" Fäle auch Ausnahmen gestatten.

Ganz besonders herrorheben will ich, daß ich die Prophylaxis auch hier für die beste Behandlung halte. Deswegen habe ich durchgesetzt, daß im Zwickaver Krüppelheim nicht nur Kinder mit rachitischen Verkrümmungen aufgenommen werden, sondern vor allem auch solche Kinder mit schwertr Rachitis, die noch keine Verkrümmungen haben, wo wir aber überzeugt sind, daß die Lebensbedingungen zu Hause s dazu kommen lassen. Die Maßnahmen unserer Prophylaxe will ich kurz angeben: Antirachitische Kost, Bestrahlungen mit Sonnen-, Quarz- und Bogenlicht, Wassage, Einueibungen, bei älteren Kindern auch Bäder, Gymnastik, Lebertran, Ossiostose usw. Ganz besonders günstig wirkt die sonnige, freie Lage unseres Krüppelheimes, welches außerhalb der Stadt, fern von Ruß und Rauch unserer Industrie und Kohlenschächte, im Westen auf einer Anhöhe in drei großen Gebäuden mit anschließendem Garten und Wald untergebracht ist.

\section{Behandlungsnorm.}

I. Rein ahwartende Allgemeinbehandlung, auf diätetischem, medikamentösem und einfach-physikalischem Wege (Massage, Bäder, Gymnastik, Bestrahlungen).

I. Geraderichtende Behandlung, selbstverständlich unter gleichzeitiger Anwendung aller unter I angegebenen Heilmittel.

1. Allmähliche Geradrichtung, wie wir sie mit unseren Korsetten, Tag. und Nachtschienen usw. ausführen. Diese Apparate wirken alle 
ausnahmslos durch vereinigte Druek- und Zugkraft. Lagerbett aus Gips orler anderem Material rechne ich nicht zu den geradrichtenden Maßnahmen, vielmehr zu den rein prophylaktischen.

2. Die einmalige und vollkonmene (radikale) Geradrichtung: Osteoklasis, Osteotomit.

Die Irt der Bebandlung wird bedingt durch den Grad der Knochenweichheit, oler besser gesagt, den Grad des Widerstandes, den ler rerkrümmte Knochen seiner Geradrichtung entgegensetzt. Gleicht sich z. B. eine Säuglingsskoliose durch einen Druck von bestimmter Stärke noch aus, so liegt große Knochenweichheit vor. Ein anderer Knochen, schon kalkhaltiger, wird demselben Druck mehr widerstehen, der Knochen wirl ,federn". Bei den sklerotischen Knochen endlich wird derselbe Druck ingendwelchen Ausgleich nicht mehr bringen. Je größer also der Ausgleichswiderstand, um so energischer müssen unsere Maßnahmen sein, um so größer die aufzuwendende Druck- und Zugleist ung unserer geradrich tenden Behandlung.

Wie prüfen wir nun die Stärke des Widerstandess am verkrümmten Knochen? Am besten, einfachsten und unschädlichsten durch den Druck unserer Hand: Aus der Größe des zum Ausgleich der Verkrümmung nötigen Handdruckes beinesse und erkenne ich Art und IIaB meines Eingriffes.

In der folgenden Zusammenstellung habe ich ver Einfachheit halber ganz grob drei Stadien der Knochenhärte angenommen: das Stadium der blühenden Rachitis, der abklingenden Rachitis, das sklerosierende Stadium.

\section{Skoliose-Kyphoskoliose.}

1. Blikhendes Stadium: Durch Hand- abwartende Behandling, Lagerbett. druck leicht ausgleichbar

2. Abklingendes Stadium: Durch sofort festes, abnehmbares Korsett. Handdruck unvollkonmen ausgleichbar

3. Sklerosiertes Stadium: Jerler Ausgleich durch Handdruck unmöglich

Gipsbehandlung (Abott) + abnehn: bares Korsett.

Die Korsetts ließ ich früher meist aus Zelluloid anfertigen, heute gewöhnlich aus Lerler', selten nach Hessing aus Stoff und Stahl.

\section{0-Beine.}

1. Blühendes Stadjum: Durch Handdruck nur bei ganz weichen Knochen ausgleichbar (federt stark!)

2. Abklingendes Stadium: Cnvollkommener Ausgleich durch Handdruck (federt wenig!)

3. Sklerosiertes Stadium: Ausgleich gänzlich unmöglich (f ed ertnicht!) sofort Schienenbehandlung, ev. Osteoklasie (Infraktion).

Osteoklasie, Schienennachbehandlung.

Osteoklasie (selten Osteotonie), er. Schienennachbehandlung. 


\section{X-Beine.}

A. Verkrümmung sitzt im Kniegelenk, das eigentliche Genu valyum (,, Gelenk-X-Bein").

1. Blühendes Stadium: Ausgleich Schienenbehandung. dur.h Handulruck leicht möglich

2. Abklingondes Stadium: Ausgleich durch Handilruck schwer oder nirlht mö̈glich

Geradrichtung und Gips + Schientennachbehandlung.

B. Verkrümnung sitzt im Knochen. Femur oder Tibia (..Knochen-X-Bein").

1. Blühendes Stadium : Leidlicher Ausgleich rlurh Handdruck (federt stark!)

2. Abklingendes Stadium: Ausgleich durch Handrtruck gering möglich (f edert wenig!)

3. Sklerosiertes Stadiun: Ausgleich gar nicht möglich (federt nicht!)
Schienenbehandlung oder Osteoklasit (Infraktion).

Osteoklasir (Schienennachbehandlung).

Osteoklasie, selten Osteotomie (meist auch hier sohienennachbehandlung, zumal dann, wenn nicht sämtliche Verkrümmungsradiusse. behoben wurden).

\section{Verkriimmungen der Arme.}

1. Blühendes Stadium: Ausgleich Schienenbehandlung. durch Handdruck leirlich möglich (federt stark!)

2. Abklingendes Stadium: Ausgleich rlureh Handriruck wenig möglich (federt wenig!)

3. Sklerosiertes stadium: Ausgleich lurch Händedruck nicht möglich (f edert nich t!)

Osterklasie (ev. Schienennachbehamllung, doch meist nicht nötig).

Osteoklasie, beinahe nie Ostertomie (Schienenbehandlung nicht nötig).

Vit diesen Behandungrsvorschlägen befinde ich mich vollkommen im Einklang mit den Thesen Hohmanns auf dem rliesjährigen Orthopädenkongrel3, nur daß ich auch in floriden Staclium ofter als er zur Osteoklasie (Infraktion) greife. 